\title{
Application of Wireless Contralateral Routing of Signal Technology in Unilateral Cochlear Implant Users with Bilateral Profound Hearing Loss
}

DOI: $10.3766 /$ jaaa. 17121

\author{
Hillary A. Snapp* \\ Michael E. Hoffer* \\ Anthony Spahr $\dagger$ \\ Suhrud Rajguru*
}

\begin{abstract}
Purpose: The aim of the study was to determine if contralateral routing of signal (CROS) technology results in improved hearing outcomes in unilateral cochlear implant $(\mathrm{Cl})$ patients and provides similar gains in speech perception in noise to traditional monaural listeners (MLs).
\end{abstract}

Research Design: The study is a prospective, within-subject repeated-measures experiment.

Study Sample: Adult, English-speaking patients with bilateral severe-profound sensorineural hearing loss using an Advanced Bionics $\mathrm{Cl}(\mathrm{n}=12)$ in one ear were enrolled for the study.

Intervention: Hearing performance in the monaural listening condition $(\mathrm{Cl}$ only) was compared with the CROS-aided (unilateral $\mathrm{Cl}+\mathrm{CROS}$ ) condition. Participants were tested for speech-in-noise performance using the Bamford-Kowal-Bench Speech-in-Noise ${ }^{T M}$ test materials in the speech front/noise front ( 0 degrees/ 0 degrees azimuth), speech front/noise back ( 0 degrees/180 degrees azimuth), speech deaf ear/noise monaural ear (90 degrees/270 degrees azimuth), and speech monaural ear/noise deaf ear (90 degrees/270 degrees azimuth) configurations. Localization error was assessed using three custom stimuli consisting of $1 / 3$ octave narrowband noises centered at 500 and $4000 \mathrm{~Hz}$ and a broadband speech stimulus. Localization stimuli were presented at random in the front hemifield by 19 speakers spatially separated by 10 degrees. Outcomes were compared with a previously described group of traditional MLs in the CROS-aided condition (normal hearing ear + CROS).

Data Collection and Analysis: All participants were tested acutely with no adaptation to the CROS device. Statistical analyses were performed using Wilcoxon signed rank tests for nonparametric data and paired sample. Statistical significance was set to $p<0.00625$ after Bonferroni adjustment for eight tests.

Results: Significant benefit was observed from unaided to the $\mathrm{Cl}+\mathrm{CROS}$-aided condition for listening in noise across most listening conditions with the greatest benefit observed in the speech deaf ear/noise monaural ear (90 degrees/270 degrees azimuth) condition $(p<0.0005)$. When compared with traditional MLs, no significant difference in decibel gain from the unaided to CROS-aided conditions was observed between participant groups. There was no improvement in localization ability in the CROS-aided condition for either participant group and no significant difference in performance between traditional MLs and unilateral $\mathrm{Cl}$ listeners.

Conclusions: These findings support that unilateral $\mathrm{Cl}$ users are capable of achieving similar gains in speech perception to that of traditional MLs with wireless CROS. These results indicate that the use of

*Department of Otolaryngology, University of Miami Ear Institute, University of Miami Miller School of Medicine, Miami, FL; †Advanced Bionics, LLC, Valencia, CA

Corresponding author: Hillary A. Snapp, Department of Otolaryngology, University of Miami Ear Institute, University of Miami Miller School of Medicine, Miami, FL 33136; Email: hsnapp@med.miami.edu

The research reported here was supported in part by Advanced Bionics, LLC. Portions of the data were presented at the 13th European Symposium Pediatric Cochlear Implants, May 2017, Lisbon, Portugal, and at the Association for Research in Otolaryngology MidWinter Meeting, February 2017, Baltimore, MD. Hillary Snapp is on the audiology advisory board for Advanced Bionics, LLC. Anthony Spahr is the Director of Audiology at Advanced Bionics, LLC. 
wireless CROS stimulation in unilateral $\mathrm{Cl}$ recipients provides increased benefit and an additional rehabilitative option for this population when bilateral implantation is not possible. The results suggest that noninvasive CROS solutions can successfully rehabilitate certain monaural listening deficits, provide improved hearing outcomes, and expand the reach of treatment in this population.

Key Words: contralateral routing of signal, listening, monaural, unilateral cochlear implant

Abbreviations: $\mathrm{Cl}=$ cochlear implant; $\mathrm{CROS}=$ contralateral routing of signal; $\mathrm{MLs}=$ monaural listeners; $\mathrm{NH}=$ normal hearing; PSNHL = profound sensorineural hearing loss; $\mathrm{SIN}=$ speech-innoise; SNR = signal-to-noise ratio

\section{INTRODUCTION}

$\mathrm{M}$ ore than $5 \%$ of the world's population, approximately 360 million people, has disabling hearing loss, yet rehabilitative devices such as hearing aids and cochlear implants (CIs) are only able to reach about $10 \%$ of the global need (Blamey et al, 2001). The deleterious effects of hearing loss are pervasive, resulting in increased disability and handicap as well as reduction in quality of life (Gatehouse and Noble, 2004; Silverman et al, 2006; Araujo et al, 2010; Sano et al, 2013). The impact of hearing loss reaches far beyond just the individual affected. According to the World Health Organization, unaddressed hearing loss costs upward of 750 billion international dollars annually (Blamey et al, 2001). CIs have the widest utility in managing bilateral profound sensorineural hearing loss (PSNHL). CIs have been demonstrated not only to be cost-effective, but also to significantly reduce the associated societal burden. Still, the initial investment required to obtain a CI is costly (Blamey et al, 2001). Despite long-term economic benefits, the high cost of these systems remains prohibitive, particularly in low-income societies (Bond et al, 2009; Lin and Albert, 2014). Although a critical mass of evidence exists in support of bilateral implantation for bilateral PSNHL (Litovsky et al, 2004; Schleich et al, 2004; van Hoesel, 2004; Litovsky et al, 2006; Grantham et al, 2007; Godar and Litovsky, 2010; Balkany, 2012), cost-benefit analysis suggests that the greatest benefit is received with unilateral implants and that effectiveness is only incrementally enhanced with bilateral implants (Summerfield et al, 2002; Lammers et al, 2011; Chen et al, 2014). Such studies are highly influential in driving health-care policy. As such, access to hearing health care around the globe limits most individuals with hearing loss to unilateral treatment.

For unilateral CI users, however, the deficits inherently associated with monaural listening remain despite the considerable benefits achieved through implantation (Litovsky et al, 2004; Schleich et al, 2004; van Hoesel, 2004; Litovsky et al, 2006; Grantham et al, 2007; Godar and Litovsky, 2010; Balkany, 2012). The association of adverse hearing outcomes with unilateral hearing impairment has, in recent years, garnered con- siderable attention by the scientific community. Studies of unilateral hearing loss have offered much insight into the deficits associated with monaural listening, most notably inability to perceive speech in background noise and localize sounds (Welsh et al, 2004; Vermeire and Van de Heyning, 2009; Wie et al, 2010; Arndt et al, 2011; Snapp, Holt, et al, 2017). This is due to the loss of auditory cues provided through binaural hearing. It has been long known that the ability to process complex auditory signals is enhanced by the ability to detect sound at the two ears. Binaural hearing provides listeners with specific interaural timing and levels cues, which are processed in the auditory cortex for interpretation of complex acoustic signals. In bilateral PSNHL, bilateral cochlear implantation has been shown to provide binaural benefit as demonstrated by improved speech perception in noise (Fetterman and Domico, 2002; Litovsky et al, 2006; Litovsky et al, 2009) and localization ability (Litovsky et al, 2006; Grantham et al, 2007; Litovsky et al, 2009). Bilaterally deafened adults who are unilaterally implanted are not able to take advantage of this potential binaural benefit.

The primary deficit for monaural listeners (MLs) is the reduced ability to hear in background noise (Giolas and Wark, 1967; Sargent et al, 2001). This effect is most significant when the signal is directed at the impaired ear and noise is masking the good ear. Although binaural processing contributes to improved listening in noise ability, the deficit incurred by MLs is largely a physiological phenomenon known as the head shadow effect. In monaural listening, the head acts as an acoustic barrier attenuating signals directed at the impaired ear by as much as $20 \mathrm{~dB}$ in the high frequencies (Middlebrooks and Green, 1991; Gilkey and Good, 1995). In MLs, loss of high-frequency cues as a result of the head shadow directly contributes to poor perception of speech, particularly in noise.

Providing bilateral input to MLs through rerouting of signal overcomes some of these disadvantages. Traditional MLs who have unilateral PSNHL with normal hearing $(\mathrm{NH})$ in the opposite ear have long benefited from devices that allow for lifting of the head shadow (Harford and Barry, 1965; Harford and Dodds, 1966; Gelfand, 1979; Niparko et al, 2003; Wazen et al, 2003; Lin et al, 2006). Studies show that contralateral routing of signal (CROS) technology, where the signal of 
interest is routed from the impaired (deaf) ear to the normal cochlea for processing, is successful in improving speech perception in noise for individuals with unilateral PSNHL (Harford and Barry, 1965; Harford and Dodds, 1966; Gelfand, 1979; Snapp, Holt, et al, 2017). Rerouting input from the impaired side to the $\mathrm{NH}$ side overcomes some of the auditory deficits experienced by MLs, suggesting that unilateral CI recipients, who in effect become MLs, may also benefit from rerouting input in the absence of binaural input. Although bilateral CI does not fully restore binaural function, improved binaural integration abilities are observed in bilateral CI recipients. Lifting of the head shadow, however, can be seen in individuals who do not necessarily demonstrate other tasks of binaural function, such as summation or localization. The same has been demonstrated in traditional MLs who use CROS hearing aids as a treatment solution for unilateral PSNHL (NH + CROS). Without any access to binaural cues, these individuals consistently demonstrate improved speech-in-noise (SIN) performance through elimination of the head shadow effect (Niparko et al, 2003; Wazen et al, 2003; Hol et al, 2004; Lin et al, 2006; Hol et al, 2010). Despite considerable gains in audibility, unilateral CI recipients continue to demonstrate poor perception of speech in background noise (Fetterman and Domico, 2002; Litovsky et al, 2006; Litovsky et al, 2009) and localization (Litovsky et al, 2006; Grantham et al, 2007; Litovsky et al, 2009), consistent with traditional MLs.

Rerouting of bilateral input for MLs is a promising alternative for patients who cannot benefit from binaural input (Arora et al, 2013; Weder et al, 2015; Taal et al, 2016). Early studies of CROS application in unilateral CI have demonstrated variable results (Arora et al, 2013; van Loon et al, 2014; Grewal et al, 2015; Guevara et al, 2015; Weder et al, 2015). Outcomes were reported in terms of hearing performance in noise. SIN measures can be either fixed where a percent correct score is obtained at a fixed signal-to-noise ratio (SNR) level, or adaptive where either the speech or the noise is adjusted to determine an individual's threshold (SNR, where $50 \%$ of the presented speech can be identified) for hearing in noise (Taylor, 2003). Studies measuring differences in overall percent correct scores suggested limited benefit (Arora et al, 2013; Grewal et al, 2015; Guevara et al, 2015), whereas studies of speech reception thresholds suggested a positive effect of CROS input to the CI (van Loon et al, 2014; Weder et al, 2015; Taal et al, 2016). Given the variability in speech perception among CI recipients, threshold measures may be more resistant to these inherent variances in the CI population. As expected (Niparko et al, 2003; Lin et al, 2006), the assessment of CROS benefit when speech is in front of the unilateral CI listener (Arora et al, 2013; Grewal et al, 2015) results in marginal benefit compared with those designed to measure head shadow effect (van Loon et al, 2014; Taal et al, 2016). Furthermore, these studies all used hearing aids (Arora et al, 2013; Grewal et al, 2015; Guevara et al, 2015; Weder et al, 2015; Taal et al, 2016) or lapel microphones (van Loon et al, 2014) hard-wired to a CI processor to deliver the contralateral microphone input. Although promising, such systems are not optimized for application in CIs, and variables such as directional microphones were not considered. Recent technological advances applied in CROS technology may improve outcomes in traditional MLs over these early hard-wired systems. However, novel CROS technology, which allows for wireless transmission of the signal, has yet to be applied to unilateral CI users.

The present study aimed to investigate the benefit of CROS input in unilateral CI recipients using a novel customized hearing device providing wireless CROS input to the implanted ear (Advanced Bionics, LLC, Valencia, CA) using measures of SIN performance and localization ability.

\section{MATERIALS AND METHODS}

\section{Participants}

University of Miami Institutional Review Board approval was obtained for this study. Individuals aged 18 years and older, who are primary English speakers, were included for the study. Enrollment was limited to postlingually deafened and experienced processor users defined as consistent users of their hearing device for at least 6 months. Twelve bilateral PSNHL patients ranging in age from 18 to 76 years (mean, 51 years \pm 18 years) using an Advanced Bionics CI in at least one ear were included for the study. Evaluation and testing was conducted using the better performing ear defined as greater than $50 \%$ word recognition ability on Consonant-Nucleus-Consonant (CNC) word testing in quiet in the aided condition. To determine if degree of CROS benefit in unilateral CI recipients was comparable with that of traditional MLs, outcomes were compared with a previously described (Snapp, Hoffer, et al, 2017) group of 12 unilateral PSNHL participants with no prior CROS hearing aid use and $\mathrm{NH}$ in the contralateral ear using a standard CROS hearing aid.

\section{Study Procedure}

This study was designed as a prospective, withinsubject repeated-measures experiment in which each participant served as his/her own control. The participants were evaluated in two phases randomized to either the monaural (CI Only) or CROS-aided (CI + CROS) conditions. Each phase consisted of two intervals with random assignment to either speech perception in noise or localization testing. All participants were allowed a minimum 15-minute break between intervals. For 
the second phase, the participants underwent testing in either the monaural (CI) or CROS (CI + CROS) conditions, alternate to phase 1 . All participants were evaluated acutely with no adaptation to the CROS condition; however, when the CROS phase was initiated, a minimum of a 15-minute acclimatization period was provided before laboratory testing.

\section{Hearing Devices}

Unilateral CI users were fitted with a customized Advanced Bionics Naida CI processor designed to wirelessly route the acoustic signal from the nonimplanted ear to the CI for the CROS condition (Figure 1). As with the traditional MLs using CROS, the CI + CROS used Hearing instrument Body Area Network for the wireless streaming of acoustic signals by transmitting digital data at a carrier frequency of $10.6 \mathrm{MHz}$ via a digital magnetic induction link from the CROS transmitter to the receiving antennae loaded within the sound processor (Wolfe and Schafer, 2015). The participants' own programs were loaded to the research processor and no changes to programming of the CI processor made before connecting the CROS microphone. The input to the CI processor was set to a 50/50 split between the CI and the wireless CROS microphone. As this is a single-participant study

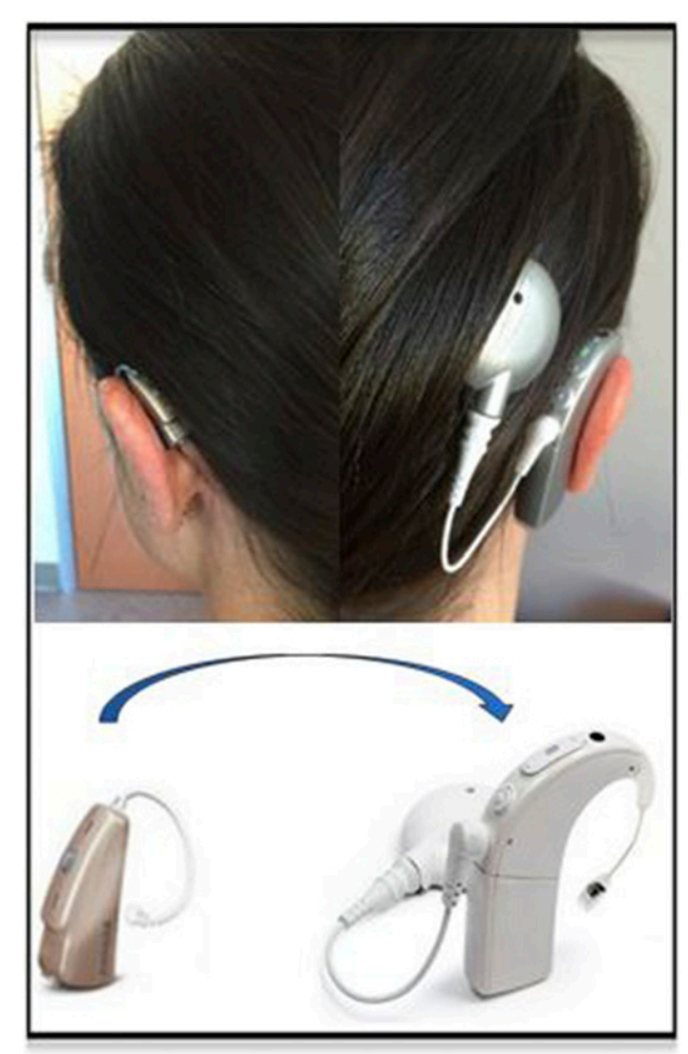

Figure 1. Customized initial CROS prototype from Advanced Bionics designed to wirelessly route the acoustic signal from the nonimplanted ear to a Naida CI processor. design, the change in performance is based on the independent variable of the CROS condition. However, the following requirements were maintained for all participants: (a) omnidirectional microphone mode and (b) CI processors were maintained in front-mic mode.

\section{Stimuli}

\section{Localization}

Stimuli were presented in a custom $4-\mathrm{m} \times 4-\mathrm{m} \times 2$-m sound booth with 19 loudspeakers with a radius of $1.3 \mathrm{~m}$ spatially separated by 10 degrees, spanning \pm 90 degrees. Localization stimuli were generated by a custom MATLAB front-end for TDT RX8 real-time multichannel processor and series of Crown Audio CT-8150 eight-channel amplifiers (Tucker-Davis Technologies, Gainesville, FL). Localization stimuli included a narrowband $350-\mathrm{msec} 1 / 3$ octave noise centered at 500 $\mathrm{Hz}$, a narrowband $350-\mathrm{msec} 1 / 3$ octave noise centered at $4 \mathrm{kHz}$, and a broadband 189-msec male-voiced "hey," band-passed from 100 to $8000 \mathrm{~Hz}$. Three trials of each stimulus were presented at $65-\mathrm{dB}$ SPL roved by $\pm 4 \mathrm{~dB}$ for a total of 171 stimuli per participant per condition. The perceived location of the sound sources was recorded using an Arduino-based 24-pushbutton feedback panel. Localization was assessed in the CI only (monaural) and CI + CROS conditions. Localization results were compared with outcomes in $\mathrm{NH}$ controls and traditional MLs with unilateral PSNHL using a CROS hearing aid (Snapp, Hoffer, et al, 2017).

\section{SIN}

Recorded commercially available BKB-SINTM sentences were used for assessment of SIN performance. The BKBSINTM uses Bamford-Kowal-Bench sentences (Bench et al, 1979) recorded in four-talker babble requiring the listener to identify key words. A minimum of two lists of 10 sentences were completed for each condition and averaged to determine the SNR level in $\mathrm{dB}$ required to obtain $50 \%$ accuracy, expressed as the SNR-50, in dB (Etymotic Research, 2005). Speech stimuli were presented at $62-\mathrm{dB}$ SPL. The test was conducted in the sound field, and the participants were evaluated in the speech front/noise front ( 0 degrees $/ 0$ degrees azimuth), speech front/noise back (0 degrees/180 degrees azimuth), speech deaf ear/noise monaural ear (90 degrees/270 degrees azimuth), and speech monaural ear/noise deaf ear (90 degrees/270 degrees azimuth) configurations (Figure 2). All test protocols were repeated in the CROS (aided) condition to characterize performance changes in this condition.

Statistical analyses were performed using Wilcoxon signed rank tests for nonparametric data and paired sample, as the outcome both on a histogram and a 


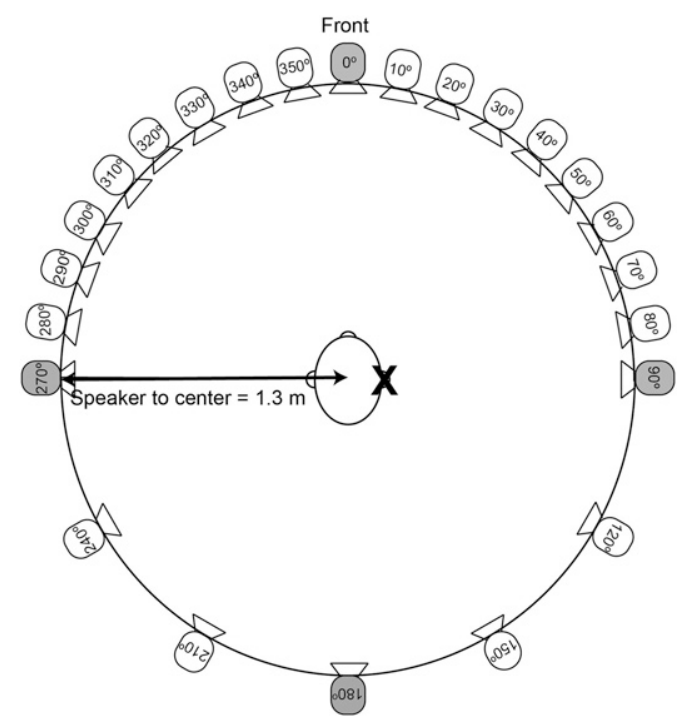

Figure 2. Diagram of the experimental setup for assessment of SIN and localization ability. Gray speakers indicate the locations for the four SIN conditions. Localization was presented in the front hemifield 90 degrees to 270 degrees.

quantile-quantile plot does not support an assumption of normality for this sample. Statistical significance was set to $p<0.00625$ assuming a type I error rate per test of 0.05 that was adjusted via a Bonferroni correction to account for eight tests. All analyses were performed using SAS JMP $^{\text {TM }}$ software (SAS Institute version 12.1; Cary, NC).

\section{RESULTS}

\section{Localization Performance}

When compared with NH listeners (Snapp, Hoffer, et al, 2017), MLs displayed high degrees of localization error (Figure 3). Unilateral CI users had comparable performance on tasks of localization to traditional MLs (Snapp, Hoffer, et al, 2017) (Table 1) with no significant difference observed between groups for any of the presented stimuli $(p>0.00625)$. The root mean square (RMS) error \pm 1 SD for each of the three localization stimuli in the CROS-aided condition is reported in Figure 3 for traditional MLs versus unilateral CI users. In the CROS-aided condition, neither the traditional MLs nor the unilateral CI users gained any localization benefit (Figure 3), and there was no difference in aided performance between groups ( $p>0.00625$ ).

\section{SIN Performance}

The median-aided CROS benefit for each of the four experimental configurations for CI + CROS users is presented against traditional MLs $(\mathrm{NH}+\mathrm{CROS})$ in Figure 4. Here, gain (improved performance) is presented as a positive change in $\mathrm{dB}$ value, whereas loss (decrease in performance) is presented as a negative change in $\mathrm{dB}$ value. Between-group analysis of overall aided benefit resulted in no statistically significant difference between traditional MLs (Snapp, Hoffer, et al, 2017) and unilateral CI users in the CROS-aided condition for any of the listening configurations $(p>0.00625$, Wilcoxon signed rank). Differences in unaided and aided performance for the CI + CROS group can be found in Figures 5A and $\mathrm{B}$ where the median absolute performance is presented as the SNR-50 or dB level of the sentences relative to the noise required by the participant to correctly repeat the BKB-SINTM sentences $50 \%$ of the time. For Figure 5, better performance corresponds with lower $\mathrm{dB}$ values. For the unilateral CI user, application of the CROS microphone resulted in significant improvement for the speech front/noise front $(p<0.0005$, median change $=+2.5$ - $\mathrm{dB}$ benefit, Wilcoxon signed rank), and speech poorer ear/noise better ear $(p<0.0005$, median change $=+9.75-\mathrm{dB}$ benefit, Wilcoxon signed rank) configurations (Figure 5A). No significant
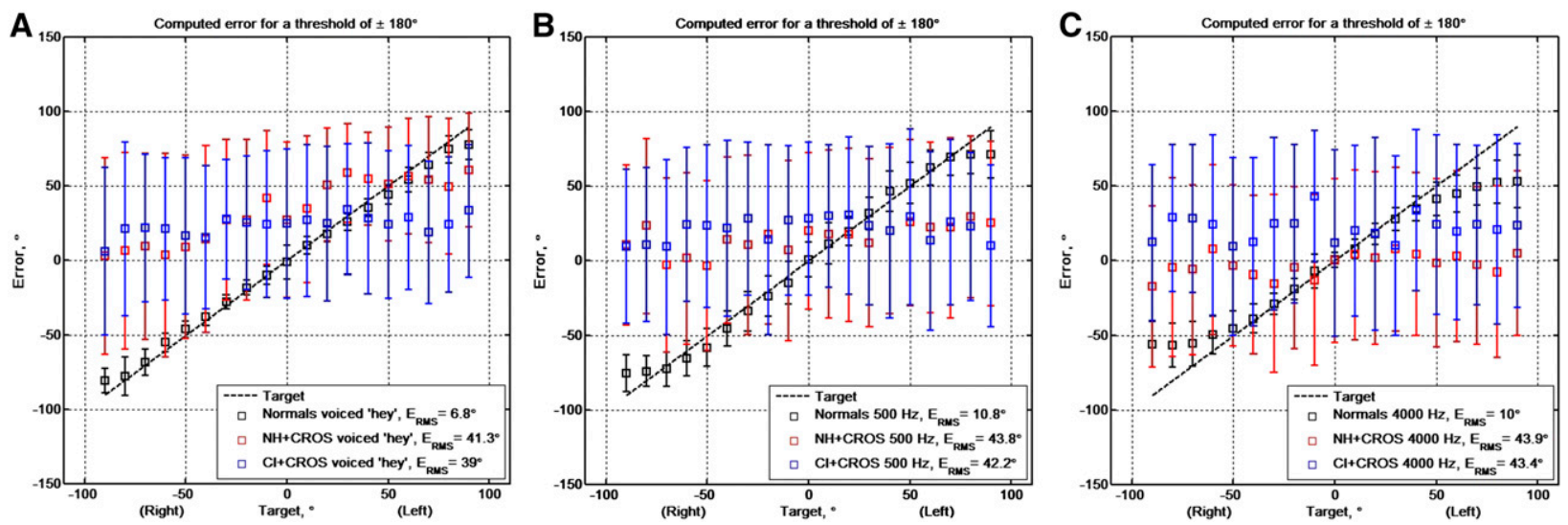

Figure 3. Aided localization performance represented in RMS error for the CI + CROS condition (blue) for the voiced "hey" (A), $500 \mathrm{~Hz}$ $1 / 3$ oct (B), and $4000 \mathrm{~Hz} 1 / 3$ oct noise (C), respectively. Aided responses are plotted against NH + CROS (red) and normal hearers (black) (Snapp, Hoffer, et al, 2017). 
Table 1. RMS Error for Three Localization Stimuli Demonstrating Unilateral $\mathrm{Cl}$ Users Have Similar Localization Performance to Traditional MLs (Snapp, Hoffer, et al, 2017)

\begin{tabular}{lccc}
\hline & Normal Hearers & MLs & Unilateral Cl \\
\hline Broadband voiced "hey" & $6.8^{\circ}$ & $41.8^{\circ}$ & $46.6^{\circ}$ \\
$1 / 3$ Octave noise $500 \mathrm{~Hz}$ & $10.8^{\circ}$ & $45.3^{\circ}$ & $48.7^{\circ}$ \\
$1 / 3$ Octave noise $4000 \mathrm{~Hz}$ & $10.0^{\circ}$ & $45.0^{\circ}$ & $43.3^{\circ}$ \\
\hline
\end{tabular}

change in performance was observed for speech front/noise back $(p>0.00625$, median change $=+1.75 \mathrm{~dB}$, Wilcoxon signed rank). A significant decrement in performance was observed when speech was directed at the CI (better ear) and noise was directed at the CROS microphone ( $p=$ 0.002 , median change $=-3.0 \mathrm{~dB}$, Wilcoxon signed rank test). Variability for unaided versus aided conditions for the CI + CROS group are presented in Figure 5B.

\section{DISCUSSION}

$\mathrm{D}$ espite considerable advancements in implantable technology and irrefutable evidence that binaural hearing is essential for advanced tasks of auditory function (Balkany et al, 1988; Litovsky et al, 2004; Nopp et al, 2004; van Hoesel, 2004; Litovsky et al, 2006; Litovsky et al, 2009; Balkany, 2012), many individuals with severe-profound bilateral hearing loss continue to have limited access to treatment (Blamey et al, 2001; Chen et al, 2014). Studies have estimated a maximum of $36 \%$ of bilateral PSNHL patients are bilateral implant recipients (Peters et al, 2010). This suggests that more than $60 \%$ of current CI recipients with bilateral PSNHL function as MLs, thereby subject to the deficits inherent to monaural listening. We sought to investigate the benefits of rerouting of signal from the impaired ear to the better ear using CROS technology of two monaural listening groups: (a) unilateral PSNHL and (b) bilateral PSNHL unilaterally implanted with a CI. Standard treatment for MLs uses the normal cochlea to deliver sound from the impaired side to the better hearing ear via CROS technology. Our data provide strong evidence that benefits gained in traditional MLs through CROS technology are achievable in unilateral CI recipients. Studies have shown that CROS technology successfully lifts the head shadow, allowing for spatial

\section{SPEECH-IN-NOISE PERFORMANCE}

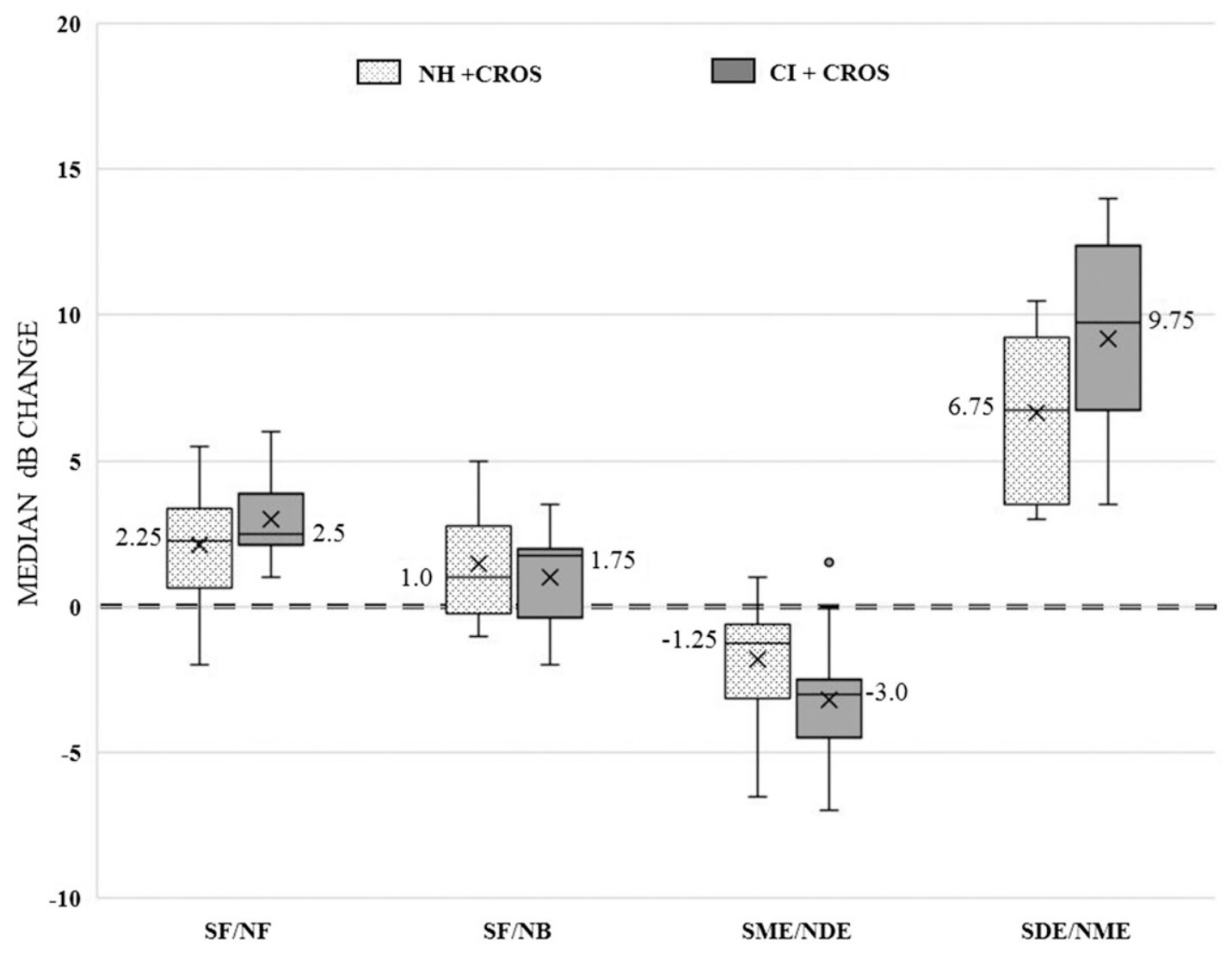

Figure 4. Aided CROS benefit for each of the four experimental configurations in traditional MLs (NH + CROS) (Snapp, Hoffer, et al, 2017) and unilateral CI (CI + CROS) groups is presented as a change in $\mathrm{dB}$ from the monaural (no CROS) condition. Here, improved performance is presented as a positive $\mathrm{dB}$ value, whereas decrease in performance is presented as a negative dB value. No significant difference between systems is observed in aided benefit or absolute performance across the listening configurations $(p>0.00625$, Wilcoxon signed-rank). 


\section{SPEECH-IN-NOISE PERCEPTION}
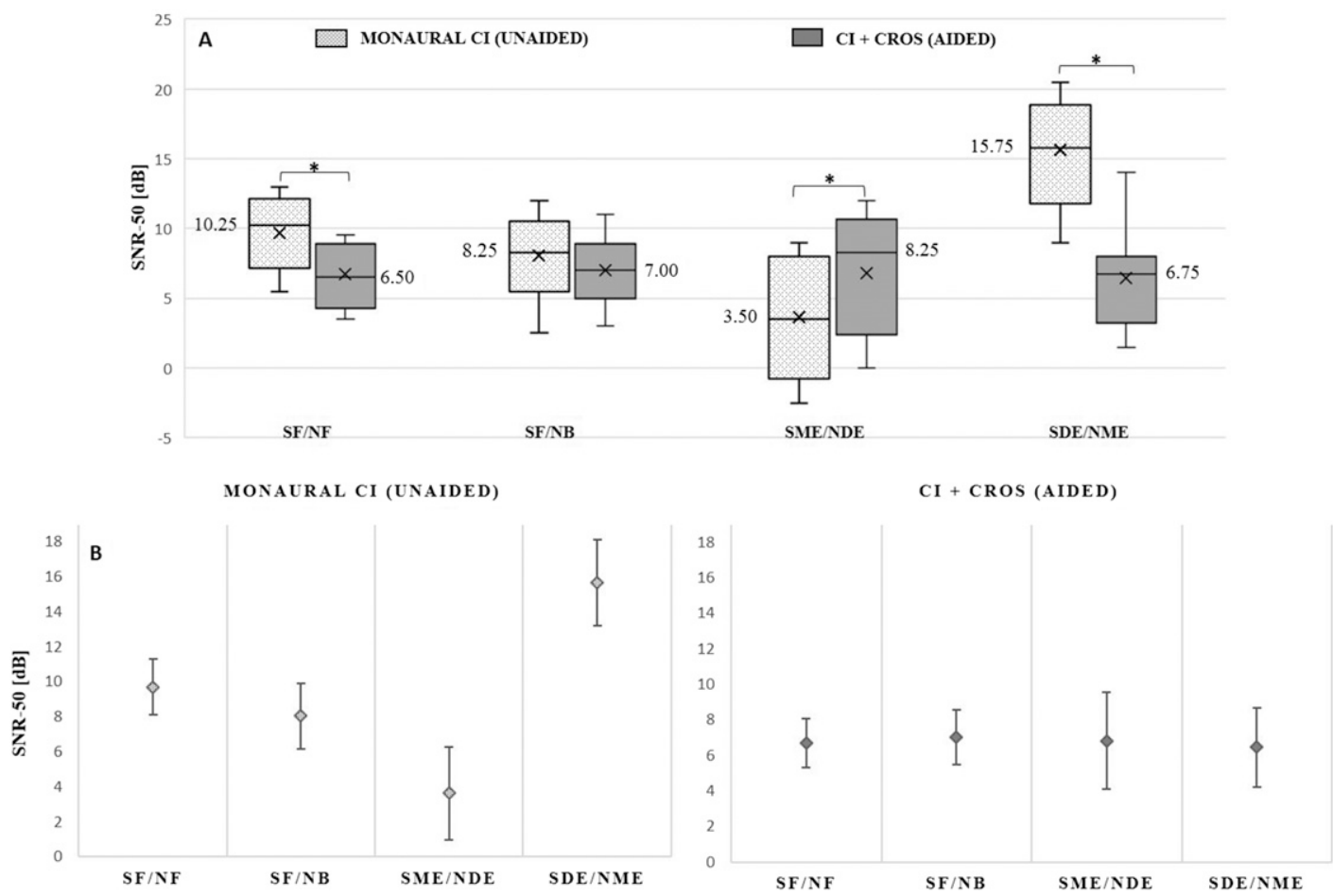

Figure 5. SNR-50 for the unaided (unilateral CI) and aided (CI + CROS) conditions for the CI group is presented in A where better performance corresponds with lower SNRs. (B) This shows the 95\% confidence intervals demonstrating variability across listening configurations for unaided (CI only) vs. CI + CROS aided.

separation of speech from background noise but is not successful in improving auditory processing abilities that require binaural inputs such as localization (Niparko et al, 2003; Lin et al, 2006; Snapp, Holt, et al, 2017). Our data are consistent with previous reports in traditional MLs, suggesting benefit from lifting of the head shadow but not in tasks requiring binaural function (Niparko et al, 2003; Lin et al, 2006; Snapp, Holt, et al, 2017).

For tasks of localization, there was no observable benefit in the horizontal plane for either traditional MLs or unilateral CI users when the CROS technology was applied. There was no significant difference between participant groups in aided performance for any of the three localization stimuli. Localization is a binaural phenomenon requiring input from two ears. As with traditional MLs (Harford and Barry, 1965), CROS microphone serves to transmit the acoustic signal arriving to the nonimplanted ear to the CI, thereby only providing unilateral input. Although individuals gain access to sounds arriving to the contralateral (nonimplanted) ear, they do not regain interaural timing and level cues provided through binaural hearing required to restore localization.

For measures of speech perception in noise, significant improvement from the unaided to aided condition for both traditional MLs and unilateral CI users was observed when speech is directed at the deaf ear and noise is masking the monaural ear, with no significant differ- ence for aided performance between participant groups. These findings demonstrate that unilateral CI users display similar deficits for listening in noise (Figure 4) and localization ability (Snapp, Hoffer, et al, 2017) (Table 1) to traditional MLs. Likewise, our findings support that unilateral CI users are capable of achieving similar gains in speech perception to that of traditional MLs with wireless CROS. Unilateral CI users' speech perception in noise ability improves across most listening conditions with the exception of noise presented to the contralateral ear. This is expected, as the CROS microphone transmits noise to the CI. Although significant, the decrement in performance is minimal $(\sim 3 \mathrm{~dB})$ in comparison to the notable gain $(\sim 9 \mathrm{~dB})$ in performance observed in the most debilitating listening condition, when speech is presented to the nonimplanted ear.

This is the first investigation of contemporary wireless CROS technology in unilateral CI users. Grewal et al (2015) investigated the potential benefits of CROS technology in unilateral CI users by coupling a hearing aid via a wired input to the CI. Their findings suggested limited benefit of CROS input to the CI. The design of this study differed in a number of ways. The investigators looked at overall percent correct for speech when presented in front of the listener. As expected, a decrease in performance was observed when noise was masking the CROS. However, they also observed a 
decrease in performance for the CROS condition when noise was lateralized to the CI. It is unclear how application of the CROS in this condition would significantly reduce performance. It is possible that the discrepancy in results is due to differing test designs. Specifically, Grewal et al (2015) used overall percent correct performance on the Arizona Biomedical Institute (AzBIO) sentence test to assess CROS benefit, whereas our study used an adaptive measure to determine the participant's threshold for hearing in noise. Given the overall low performance reported by the authors in this condition ( $45 \%$ correct), it is possible that the change of $10 \%$ as reported by the authors is simply a factor of test-retest. The present study looked to identify the SNR-50 with a lower $\mathrm{dB}$ representing better performance. Review of Figure 5 suggests that even though unilateral CI users improve in most situations with CROS input, they still consistently require a +6 -dB SNR to achieve $50 \%$ correct on measures of speech perception in noise regardless of the listening configuration. Grewal et al (2015), assessed speech perception in noise at a fixed $+5-\mathrm{dB}$ SNR for all listening configurations. It is possible that this condition $(+5 \mathrm{SNR})$ is below the threshold for which the CROS benefit may be observed in the unilateral CI user. In addition, the present study required participants to demonstrate a greater threshold of speech perception performance before enrollment, which may also account in part for some of the variance in findings.

Taal et al (2016) sought to quantify the effect of CROS for listening in unilateral CI users as function of speaker location. In accordance with the present findings, they concluded that use of a CROS results in a maximum gain in speech reception threshold of $7.9 \mathrm{~dB}$ when speech comes from the CROS side compared with a maximum loss in speech reception threshold of $2.1 \mathrm{~dB}$ when speech comes from the implanted side in the presence of diffuse noise. A model of speech intelligibility suggested that in the case of directional noise, the effect of the CROS is symmetric and the maximum loss or gain in speech reception threshold was around $9 \mathrm{~dB}$. Our findings were consistent with the predicted gain of $9 \mathrm{~dB}$ for speech directed at the CROS but only found an approximate decrement of $3 \mathrm{~dB}$ when noise was directed at the CROS. Weder et al (2015) investigation of wired CROS input via hearing aid to the CI also reported this contrast in performance. Results of traditional MLs under the CROS condition paralleled that of CI + CROS (Figure 4). Similarly, Lin et al (2006) demonstrated in $\mathrm{NH}+\mathrm{CROS}$ that the advantages of reducing the head shadow for speech directed at the better ear outweigh the disadvantages inherent in head shadow resulting from introducing noise to the impaired ear. The subjective cost-benefit of CROS input in real-world listening scenarios for unilateral CI users has yet to be studied. It is possible that transmission of noise from the CROS device to the CI will result in aversion to CI + CROS input.

Arguably, the most interesting finding is not in the benefit gained or lost, but in the absolute performance across listening conditions. Review of Figures $5 \mathrm{~A}$ and $\mathrm{B}$ demonstrates a high degree of variability in performance in the unaided condition as a function of speaker and masker location. These results suggest that when the listener is reliant on the unilateral input via the CI alone, they must continually adapt or modify their environment to improve the SNR to allow for improved speech perception. A listener would need to adjust their positioning, so the better hearing ear, in this case the CI ear, is always directed at the speaker allowing for the most optimal hearing condition. This may not be realistic in highly adaptive listening situations or in those who require sustained listening. Addition of the CROS microphone clearly reduces this variability and allows equal performance across all listening conditions regardless of the listening configuration (Figures 5A and $\mathrm{B}$ ). Although a small decrement in performance is observed when the noise is sent from the CROS microphone to the $\mathrm{CI}$, it is questionable whether or not the listener is even aware of this negative effect. Consider the ML engaged in group discussion in a noisy environment who must continually adjust their head position to remain engaged in the conversation. The CROS user, on the other hand, has consistent speech perception performance without having to adjust to the speaker. van Loon et al (2014) compared bilateral CI users to unilateral CI users with CROS and demonstrated similar results. Although they concluded that bilateral CI resulted in the greatest degree of benefit, a significant improvement of $>6 \mathrm{~dB}$ was obtained in the CI + CROS group for speech directed at the impaired ear. Like our observation, the unilateral CI users displayed highly variable speech reception thresholds as a function of location of the speaker, with performance equalizing across the three listening conditions for CI + CROS (van Loon et al, 2014). Collectively, these observations raise intriguing questions regarding the limitations of MLs and suggest that a reduction in listening effort may be an additional benefit of the CROS. Significant gains in speech perception in noise have the potential to positively impact unilateral CI patients in substantial ways. The ability to process complex auditory signals is essential for effective communication and daily acts of living. Likewise, the inability to process these signals results in considerable reduction of quality of life, social exclusion, vocational limitations, and a high disability and handicap index (Gatehouse and Noble, 2004; Araujo et al, 2010; Augustine et al, 2013; Sano et al, 2013).

The primary limitation of this study is that all participants were tested acutely, precluding any adaptation to the CROS hearing modality. However, all CI participants were experienced users and the CI processor was set to a 50/50 split between the CI and the wireless CROS microphone. The resulting input is not novel as no changes to the processing of the signal occurred, only the addition of the CROS microphone input. The 
wireless CROS prototype (Advanced Bionics, LLC) was limited to the omnidirectional mode. As demonstrated here, unilateral CI users require increased SNRs compared with normal hearers. The potential for even greater CI + CROS benefit with the application of directional microphone technology warrants further study. Our data suggest that MLs must develop listening strategies to improve the SNR as the location of the speaker changes. With CROS input, these strategies are undoubtedly modified, requiring some adaptation for real-world listening in MLs. One such example is listening in diffuse noise environments, which was not studied here. The addition of the CROS in unilateral CI reduced the variability in performance, allowing for equal performance across all listening conditions regardless of the location of the speaker or noise. Although wireless CROS technology is a promising solution, it is unclear if unilateral CI recipients will adopt and accept CROS as a treatment option. CI + CROS does not provide binaural stimulation, or the improved performance on tasks requiring these inputs that can be achieved through bilateral CI implantation. Although CI + CROS does not replace true bilateral hearing, it could serve as an option for those who cannot obtain a second implant for insurance purposes, health reasons, etc. Although many unilateral CI recipients do not have access to a second implant (Blamey et al, 2001; Bond et al, 2009; Chen et al, 2014), there are those individuals who simply choose not to pursue a second implant. Questions remain regarding the hearing handicap in unilateral CI users. CI + CROS may offer a bridge to bilateral implantation in those individuals who have yet to realize the benefit of bilateral input. It is also possible that the decrease in performance that occurs when noise is directed to the CROS may result in poor acceptance of CROS technology in the unilateral CI population. Future studies will focus on investigating the adoption and acceptance of wireless CROS technology for unilateral CI recipients through chronic studies of objective and subjective CROS performance.

\section{CONCLUSIONS}

$\mathrm{H}$ earing loss is a global public health issue. The economic burden of hearing loss weighs heavily on both the individual and society. Yet, access to treatment continues to be limited, only serving about $10 \%$ of the global need (Blamey et al, 2001). In countries with a low per capital gross national income, the principal barrier to acquiring a hearing device is cost (Olusanya, 2009). Bilateral CIs have improved outcomes over unilateral CIs, specifically for tasks that require binaural input such as localization (Litovsky et al, 2004; Litovsky et al, 2006; Balkany, 2012; Lammers et al, 2014). However, convincing evidence that the benefit provided by adding the second implant warrants the associated cost is lack- ing (Kral and O'Donoghue, 2010). Considering these factors, it is unsurprising that those individuals who have access to CIs are often limited to a single implant. With the continued rise in health-care cost constraints, the number of patients who will not have access to binaural processing through bilateral implantation is expected to increase (Chen et al, 2014). When applied to unilateral CI users, this novel approach can overcome a key limitation of current treatment by restoring access to sound from the nonimplanted side and improve speech perception in noise. Although tasks requiring binaural input, such as localization, are not improved with CI + CROS, the access to sound awareness on the unimplanted side with wireless CROS is encouraging. The results of this study suggest that wireless CROS decreases the functional deficits for listening in noise. Wireless CROS offers an innovative approach to resolving one of the auditory deficits associated with monaural listening by lifting the head shadow effect. Furthermore, it provides a noninvasive alternative for patients who are unable or unwilling to undergo a second surgery, thereby expanding access to rehabilitation in this population.

\section{REFERENCES}

Araujo PG, Mondelli MF, Lauris JR, Richieri-Costa A, Feniman MR. (2010) Assessment of the auditory handicap in adults with unilateral hearing loss. Braz J Otorhinolaryngol 76: 378-383.

Arndt S, Laszig R, Aschendorff A, Beck R, Schild C, Hassepass F, Ihorst G, Kroeger S, Kirchem P, Wesarg T. (2011) [Unilateral deafness and cochlear implantation: audiological diagnostic evaluation and outcomes]. HNO 59:437-446.

Arora R, Amoodi H, Stewart S, Friesen L, Lin V, Nedzelski J, Chen J. (2013) The addition of a contralateral routing of signals microphone to a unilateral cochlear implant system-a prospective study in speech outcomes. Laryngoscope 123:746-751.

Augustine AM, Chrysolyte SB, Thenmozhi K, Rupa V. (2013) Assessment of auditory and psychosocial handicap associated with unilateral hearing loss among Indian patients. Indian J Otolaryngol Head Neck Surg 65:120-125.

Balkany T, Boggess W, Dinner B. (1988) Binaural cochlear implantation: comparison of $3 \mathrm{M} /$ House and Nucleus 22 devices with evidence of sensory integration. Laryngoscope 98:1040-1043.

Balkany TJ. (2012) Two cochlear implants are better than one. $J$ Pediatr 161:169.

Bench J, Kowal A, Bamford J. (1979) The BKB (Bamford-KowalBench) sentence lists for partially-hearing children. $B r J$ Audiol 13:108-112.

Blamey PJ, Sarant JZ, Paatsch LE, Barry JG, Bow CP, Wales RJ, Wright M, Psarros C, Rattigan K, Tooher R. (2001) Relationships among speech perception, production, language, hearing loss, and age in children with impaired hearing. J Speech Lang Hear Res 44: 264-285.

Bond M, Mealing S, Anderson R, Elston J, Weiner G, Taylor RS, Hoyle M, Liu Z, Price A, Stein K. (2009) The effectiveness and costeffectiveness of cochlear implants for severe to profound deafness 
in children and adults: a systematic review and economic model. Health Technol Assess 13:1-330.

Chen JM, Amoodi H, Mittmann N. (2014) Cost-utility analysis of bilateral cochlear implantation in adults: a health economic assessment from the perspective of a publicly funded program. $\mathrm{La}$ ryngoscope 124:1452-1458.

Etymotic Research. (2005) Bamford-Kowal-Bench Speech-InNoise Test (Version 1.03) [Audio CD]. Elk Grove Village, IL.

Fetterman BL, Domico EH. (2002) Speech recognition in background noise of cochlear implant patients. Otolaryngol Head Neck Surg 126:257-263.

Gatehouse S, Noble W. (2004) The speech, spatial and qualities of hearing scale (SSQ). Int $J$ Audiol 43:85-99.

Gelfand SA. (1979) Usage of CROS hearing aids by unilaterally deaf patients. Arch Otolaryngol 105:328-332.

Gilkey RH, Good MD. (1995) Effects of frequency on free-field masking. Hum Factors 37:835-843.

Giolas TG, Wark DJ. (1967) Communication problems associated with unilateral hearing loss. J Speech Hear Disord 32:336-343.

Godar SP, Litovsky RY. (2010) Experience with bilateral cochlear implants improves sound localization acuity in children. Otol Neurotol 31:1287-1292.

Grantham DW, Ashmead DH, Ricketts TA, Labadie RF, Haynes DS. (2007) Horizontal-plane localization of noise and speech signals by postlingually deafened adults fitted with bilateral cochlear implants. Ear Hear 28:524-541.

Grewal AS, Kuthubutheen J, Smilsky K, Nedzelski JM, Chen JM, Friesen L, Lin VY. (2015) The role of a new contralateral routing of signal microphone in established unilateral cochlear implant recipients. Laryngoscope 125:197-202.

Guevara N, Grech C, Gahide I, Gallego S. (2015) Assessment of the contralateral routing of signal system in unilateral cochlear implantation. Clin Otolaryngol 40:535-544.

Harford E, Barry J. (1965) A rehabilitative approach to the problem of unilateral hearing impairment: the contralateral routing of signals CROS. J Speech Hear Disord 30:121-138.

Harford E, Dodds E. (1966) The clinical application of CROS. A hearing aid for unilateral deafness. Arch Otolaryngol 83: $455-464$

Hol MK, Bosman AJ, Snik AF, Mylanus EA, Cremers CW. (2004) Bone-anchored hearing aid in unilateral inner ear deafness: a study of 20 patients. Audiol Neurootol 9:274-281.

Hol MK, Kunst SJ, Snik AF, Bosman AJ, Mylanus EA, Cremers CW. (2010) Bone-anchored hearing aids in patients with acquired and congenital unilateral inner ear deafness (Baha CROS): clinical evaluation of 56 cases. Ann Otol Rhinol Laryngol 119: $447-454$.

Kral A, O'Donoghue GM. (2010) Profound deafness in childhood. $N$ Engl J Med 363:1438-1450.

Lammers MJ, Grolman W, Smulders YE, Rovers MM. (2011) The cost-utility of bilateral cochlear implantation: a systematic review. Laryngoscope 121:2604-2609.

Lammers MJ, Venekamp RP, Grolman W, van der Heijden GJ. (2014) Bilateral cochlear implantation in children and the impact of the inter-implant interval. Laryngoscope 124:993-999.
Lin FR, Albert M. (2014) Hearing loss and dementia-who is listening? Aging Ment Health 18:671-673.

Lin LM, Bowditch S, Anderson MJ, May B, Cox KM, Niparko JK. (2006) Amplification in the rehabilitation of unilateral deafness: speech in noise and directional hearing effects with bone-anchored hearing and contralateral routing of signal amplification. Otol Neurotol 27:172-182.

Litovsky RY, Parkinson A, Arcaroli J, Peters R, Lake J, Johnstone P, Yu G. (2004) Bilateral cochlear implants in adults and children. Arch Otolaryngol Head Neck Surg 130:648-655.

Litovsky RY, Johnstone PM, Godar SP. (2006) Benefits of bilateral cochlear implants and/or hearing aids in children. Int $J$ Audiol 45: S78-S91.

Litovsky RY, Parkinson A, Arcaroli J. (2009) Spatial hearing and speech intelligibility in bilateral cochlear implant users. Ear Hear 30:419-431.

Middlebrooks JC, Green DM. (1991) Sound localization by human listeners. Annu Rev Psychol 42:135-159.

Niparko JK, Cox KM, Lustig LR. (2003) Comparison of the bone anchored hearing aid implantable hearing device with contralateral routing of offside signal amplification in the rehabilitation of unilateral deafness. Otol Neurotol 24:73-78.

Nopp P, Schleich P, D'Haese P. (2004) Sound localization in bilateral users of MED-EL COMBI 40/40+ cochlear implants. Ear Hear $25: 205-214$

Olusanya BO. (2009) Comment on Baltussen and Smith. Costeffectiveness of selected interventions for hearing impairment in Africa and Asia: a mathematical modelling approach. Int $J$ Audiol 48:159-160; discussion 160.

Peters BR, Wyss J, Manrique M. (2010) Worldwide trends in bilateral cochlear implantation. Laryngoscope 120:S17-S44.

Sano H, Okamoto M, Ohhashi K, Iwasaki S, Ogawa K. (2013) Quality of life reported by patients with idiopathic sudden sensorineural hearing loss. Otol Neurotol 34:36-40.

Sargent EW, Herrmann B, Hollenbeak CS, Bankaitis AE. (2001) The minimum speech test battery in profound unilateral hearing loss. Otol Neurotol 22:480-486.

Schleich P, Nopp P, D'Haese P. (2004) Head shadow, squelch, and summation effects in bilateral users of the MED-EL COMBI 40/40+ cochlear implant. Ear Hear 25:197-204.

Silverman CA, Silman S, Emmer MB, Schoepflin JR, Lutolf JJ. (2006) Auditory deprivation in adults with asymmetric, sensorineural hearing impairment. J Am Acad Audiol 17:747-762.

Snapp HA, Hoffer ME, Liu X, Rajguru SM. (2017) Effectiveness in rehabilitation of current wireless CROS technology in experienced bone-anchored implant users. Otol Neurotol 38:1397-1404.

Snapp HA, Holt FD, Liu X, Rajguru SM. (2017) Comparison of speech-in-noise and localization benefits in unilateral hearing loss subjects using contralateral routing of signal hearing aids or boneanchored implants. Otol Neurotol 38:11-18.

Summerfield AQ, Marshall DH, Barton GR, Bloor KE. (2002) A cost-utility scenario analysis of bilateral cochlear implantation. Arch Otolaryngol Head Neck Surg 128:1255-1262.

Taal CH, van Barneveld DC, Soede W, Briaire JJ, Frijns JH. (2016) Benefit of contralateral routing of signals for unilateral cochlear implant users. J Acoust Soc Am 140:393. 
Taylor B. (2003) Speech-in-noise tests: how and why to include them in your basic test battery. Hear $J$ 56:40, 42-46.

van Hoesel RJ. (2004) Exploring the benefits of bilateral cochlear implants. Audiol Neurootol 9:234-246.

van Loon MC, Goverts ST, Merkus P, Hensen EF, Smits C. (2014) The addition of a contralateral microphone for unilateral cochlear implant users: not an alternative for bilateral cochlear implantation. Otol Neurotol 35:e233-e239.

Vermeire K, Van de Heyning P. (2009) Binaural hearing after cochlear implantation in subjects with unilateral sensorineural deafness and tinnitus. Audiol Neurootol 14:163-171.

Wazen JJ, Spitzer JB, Ghossaini SN, Fayad JN, Niparko JK, Cox K, Brackmann DE, Soli SD. (2003) Transcranial contralateral cochlear stimulation in unilateral deafness. Otolaryngol Head Neck Surg 129:248-254.

Weder S, Kompis M, Caversaccio M, Stieger C. (2015) Benefit of a contralateral routing of signal device for unilateral cochlear implant users. Audiol Neurootol 20:73-80.

Welsh LW, Welsh JJ, Rosen LF, Dragonette JE. (2004) Functional impairments due to unilateral deafness. Ann Otol Rhinol Laryngol 113:987-993.

Wie OB, Pripp AH, Tvete O. (2010) Unilateral deafness in adults: effects on communication and social interaction. Ann Otol Rhinol Laryngol 119:772-781.

Wolfe J, Schafer E. (2015) Programming Cochlear Implants. 2nd ed. San Diego, CA: Plural Publishing. 